\title{
Does Gait Kinematic Parameters Change as Functional Outcome Scales in Total Hip Arthroplasty Subjects after Rehabilitation?
}

\author{
Pollet J1, Arienti $C^{1}$, Previcini $V^{2}$, Amici $C^{2}$, Piovanelli $B^{1}$, Buraschi $B^{1}$, Negrini $S^{1,2}$ \\ ${ }^{1}$ IRCCS Don Carlo Gnocchi Foundation, Milan, Italy; ${ }^{2}$ University of Brescia, Brescia, Italy
}

\section{Introduction}

Total hip arthroplasty (THA), is a routine, common surgical intervention; following surgery is usual practice a period of rehabilitation to recover strength, range of motion and walking ability of the operated limb. Many outcome scales (OS) have been developed to assess the improvements of the patients, but gait parameters have not been studied as possible outcome[1].

\section{Research Question}

This study aims to evaluate the effectiveness of rehabilitation through oS and gait analysis (GA) parameters, secondary to compare if the improvements detected by OS are related with GA parameters.

\section{Methods}

Subjects were recruited in a rehabilitation unit after receiving THA. Inclusion criteria were stable clinical conditions, and no other neurological or rheumatic pathologies or previous surgeries at the lower limbs. For the GA, a BTS $^{\circledR}$ DX-400 system with 8 optoelectronic cameras and 2 force platforms were used. The analysis was assessed by an expert operator.

Outcomes considered:

-Merle D’Aubigne

-Barthel Index

-Pain NRS

Gait analysis parameters considered:

-Spatio-Temporal parameters

- Gait Variable Score (GVS) [2]

-Gait Profile Score (GPS) [2]

-Gait Deviation Index (GDI) [3]

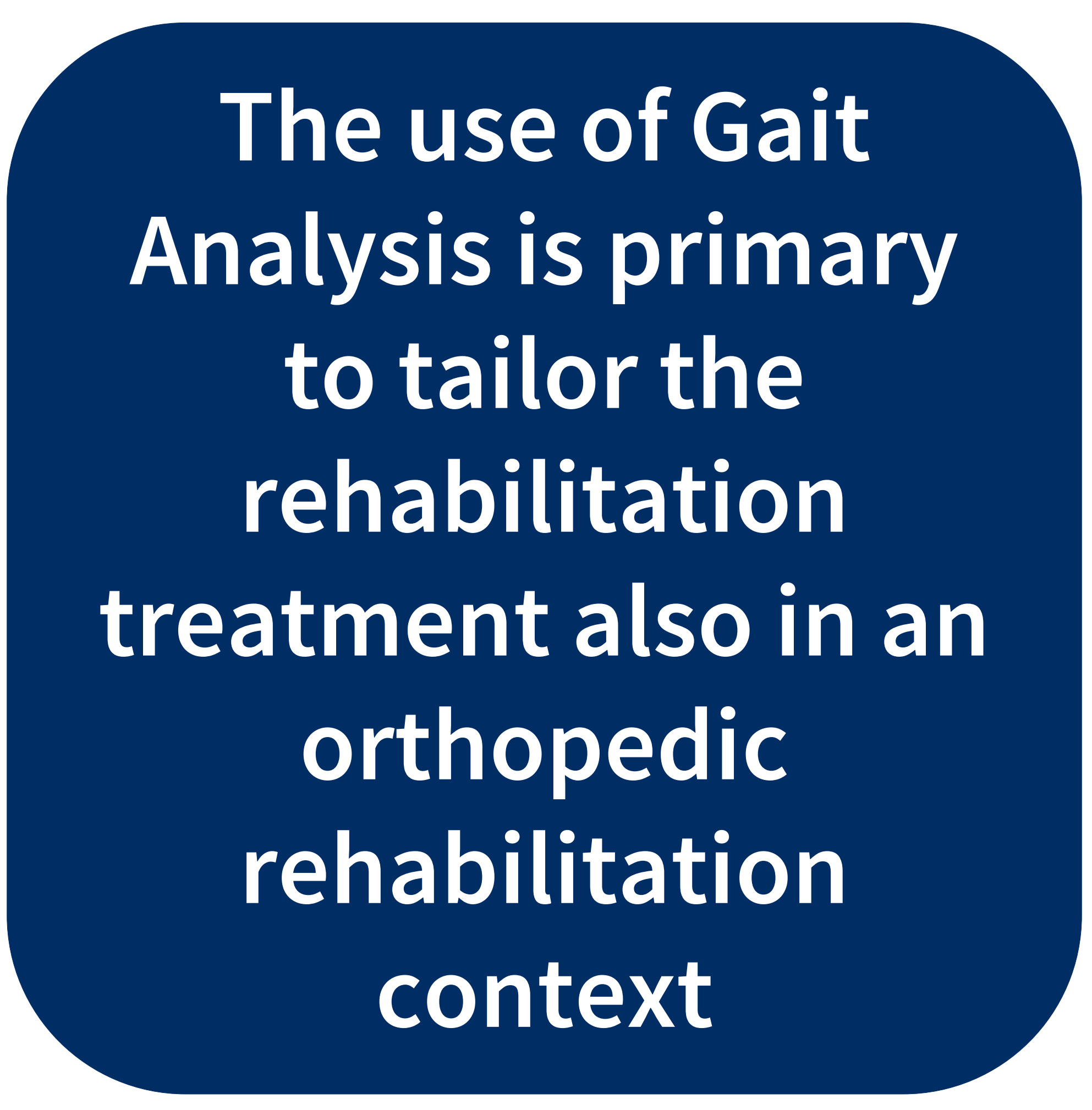

\begin{tabular}{|l|c|c|c|}
\hline $\begin{array}{l}\text { Functional Outcome } \\
\text { Scales }\end{array}$ & Admission (T0) & Discharge (T1) & pValue \\
\hline BI & $66.58 \pm 8.96$ & $94.25 \pm 6.30$ & $<0.001$ \\
\hline MDA & $7.75 \pm 1.22$ & $12.25 \pm 1.54$ & $<0.001$ \\
\hline NRS & $1.17 \pm 1.75$ & $0 \pm 0$ & 0.036 \\
\hline
\end{tabular}

\begin{tabular}{|l|c|c|c|}
\hline $\begin{array}{l}\text { Spatio-Temporal } \\
\text { Parameters }\end{array}$ & Admission (T0) & Discharge (T1) & pValue \\
\hline Speed $(\mathrm{m} / \mathrm{s})$ & $0.37 \pm 0.18$ & $0.58 \pm 0.16$ & 0.006 \\
\hline Cadence $(\mathrm{step} / \mathrm{min})$ & $60.81 \pm 19.20$ & $78.53 \pm 12.88$ & 0.014 \\
\hline Step Length $(\mathrm{m})$ & $0.61 \pm 0.24$ & $0.71 \pm 0.28$ & 0.355 \\
\hline Stride Length $(\mathrm{m})$ & $0.50 \pm 0.23$ & $0.61 \pm 0.25$ & 0.266 \\
\hline Stance phase & $70.29 \pm 8.03$ & $64.02 \pm 4.46$ & 0.027 \\
\hline Swing phase & $32.48 \pm 9.48$ & $37.55 \pm 3.93$ & 0.101 \\
\hline Single-Stance Phase & $32.31 \pm 9.58$ & $38.43 \pm 4.75$ & 0.060 \\
\hline Double-Stance Phase & $15.22 \pm 4.70$ & $13.52 \pm 3.70$ & 0.336 \\
\hline
\end{tabular}

\begin{tabular}{|l|c|c|c|}
\hline Kinematic Indexes & Admission (T0) & Discharge (T1) & pValue \\
\hline GVS Pelvic Tilt & $8.34 \pm 5.08$ & $6.79 \pm 3.92$ & 0.176 \\
\hline GVS Pelvic Rot & $5.49 \pm 2.64$ & $4.76 \pm 2.32$ & 0.426 \\
\hline GVS Pelvic Obl & $3.91 \pm 2.08$ & $3.41 \pm 1.58$ & 0.204 \\
\hline GVS Hip FE & $26.38 \pm 43.79$ & $20.93 \pm 37.28$ & 0.027 \\
\hline GVS Hip Abd-Add & $9.57 \pm 10.94$ & $9.15 \pm 10.34$ & 0.519 \\
\hline GVS Hip IE & $15.10 \pm 23.91$ & $20.73 \pm 24.57$ & 0.042 \\
\hline GVS Knee FE & $24.48 \pm 30.84$ & $21.43 \pm 30.72$ & 0.042 \\
\hline GVS Ankle FE & $12.43 \pm 2.09$ & $10.08 \pm 3.27$ & 0.003 \\
\hline GVS Ankle IE & $6.78 \pm 2.11$ & $6.63 \pm 3.76$ & 0.470 \\
\hline GPS & $15.81 \pm 19.07$ & $14.70 \pm 17.55$ & 0.380 \\
\hline GDI & $67.01 \pm 30.81$ & $72.42 \pm 27.16$ & 0.977 \\
\hline
\end{tabular}

For the statistical analysis a Student $\mathrm{T}$ and Wilcoxon signed ranks test were performed to test the variation between T0 and T1. Spearman's Rho and Pearson's R were calculated to investigate a possible relationship between $\mathrm{BI}$ and MDA, with the gait analysis parameters.

\section{Results}

12 subjects ( 4 females, 9 left hips) mean age $68 \pm 8$ were included, the length of recovery was $17 \pm 6$ days.

\section{Discussion}

Both OS and temporal and spatial GA parameters showed meaningful improvements between T0 and $\mathrm{T} 1$; these improvements have been clearly detected also with GA, that has rarely been performed before in such an acute phase[4]. The correlation between the OS and the aforementioned GA parameters, may suggest a wider use of $\mathrm{GA}$ in this clinical context, as a useful tool to assess the function and improvements of this kind of population. In particular to tailor the rehabilitation treatment.

\section{References}

[1] S. Zhao, Y. Chen, X. Zhang, Clinical application of gait analysis in hip arthroplasty, Orthop. Surg. 2 (2010) 94-99. doi:10.1111/j.17577861.2010.00070.x.

[2] R. Baker, J.L. McGinley, M.H. Schwartz, S. Beynon, A. Rozumalski, H.K. Graham, O. Tirosh, The gait profile score and movement analysis profile, Gait Posture. 30 (2009) 265-269. doi:10.1016/j.gaitpost.2009.05.020;

[3] M.H. Schwartz, A. Rozumalski, The Gait Deviation Index: a new comprehensive index of gait pathology, Gait Posture. 28 (2008) $351-$ 357. doi:10.1016/j.gaitpost.2008.05.001;

[4] J. Pollet, C. Arienti, F. Bosio, B. Piovanelli, R. Buraschi, P. Pedersini, S. Negrini, P 165 - Changes in gait kinematic parameters after rehabilitation in total knee arthroplasty subjects: A prospective observational pilot study, Gait Posture. 65 (2018) $513-514$. doi:10.1016/j.gaitpost.2018.07.086.
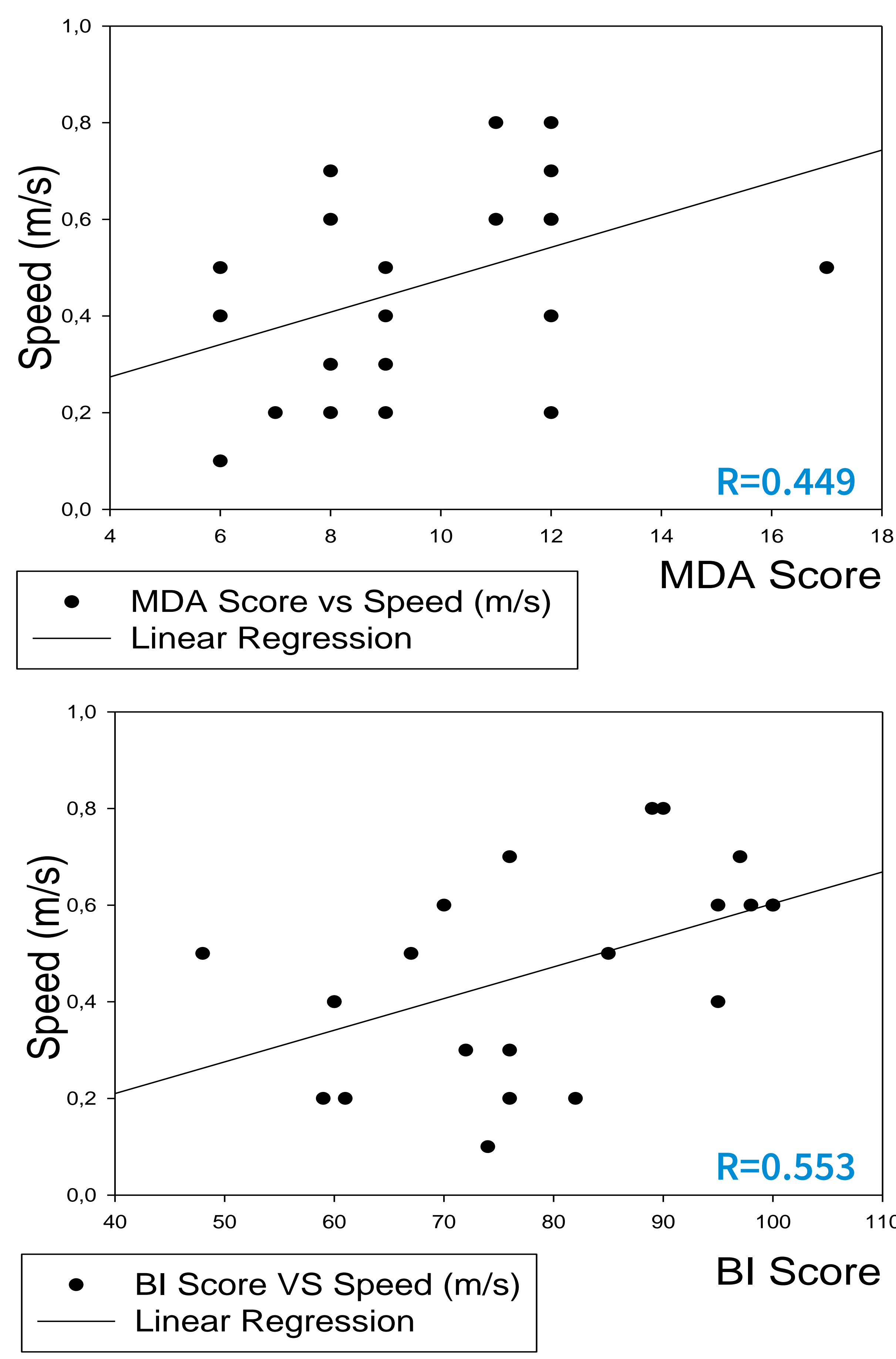

For more contact: joel.pollet.ft@gmail.comit 